PROCEEDINGS OF THE

AMERICAN MATHEMATICAL SOCIETY

Volume 132, Number 10, Pages 3055-3067

S 0002-9939(04)07400-3

Article electronically published on June 2, 2004

\title{
ASPECTS OF COMPACT QUANTUM GROUP THEORY
}

\author{
G. J. MURPHY AND L. TUSET
}

(Communicated by David R. Larson)

\begin{abstract}
We show that if a compact quantum semigroup satisfies certain weak cancellation laws, then it admits a Haar measure, and using this we show that it is a compact quantum group. Thus, we obtain a new characterization of a compact quantum group. We also give a necessary and sufficient algebraic condition for the Haar measure of a compact quantum group to be faithful, in the case that its coordinate $C^{*}$-algebra is exact. A representation is given for the linear dual of the Hopf $*$-algebra of a compact quantum group, and a functional calculus for unbounded linear functionals is derived.
\end{abstract}

\section{INTRODUCTION}

The theory of quantum groups had its origin in attempts to extend Pontryagin's duality theory for abelian locally compact groups to the nonabelian setting. Since the dual of a nonabelian group is not itself a group, it is necessary to find a larger category that includes both the groups and their duals. Pioneering work in this direction was done by G. I. Kac, M. Takesaki, M. Enock and J.-M. Schwartz; see [6] and the references therein. However, it became apparent in the 1980s that the then current theories did not encompass new and important examples due to S. L. Woronowicz [14], V. G. Drinfeld [5] and others that clearly deserved to be considered as quantum groups. A number of radically different approaches to the theory of quantum groups developed at this time. The approach most relevant for this paper is that originated by Woronowicz [13, 15] who developed the subject in terms of $C^{*}$-algebras (in the Kac algebra theory [6] von Neumann algebras are used).

A compact group is a compact space with a continuous multiplication satisfying certain extra conditions. In Woronowicz's quantum group theory, one replaces the compact space by a noncommutative, unital $C^{*}$-algebra and the group multiplication by a co-multiplication on the $C^{*}$-algebra satisfying certain conditions. In this paper we introduce new axioms for a compact quantum group that are equivalent to Woronowicz's. Our axioms - certain weak cancellation laws - are first shown to be sufficient to ensure the existence of a Haar measure and then, using this, we deduce the equivalence of these axioms and those of Woronowicz. The existence proof for the Haar measure that we give builds on A. Van Daele's simplification of Woronowicz's original proof [11], but we present some further simplifications. We

Received by the editors December 10, 2001 and, in revised form, June 3, 2003.

2000 Mathematics Subject Classification. Primary 46L89, 58B32.

Key words and phrases. Quantum group, Haar measure. 
also show, quite separately and by new and quite different methods, that an abelian compact quantum semigroup always admits a Haar measure.

We give an algebraic condition on the coordinate $C^{*}$-algebra of a compact quantum group that ensures faithfulness of the Haar measure, in the case that the $C^{*}$-algebra is exact (in particular, in the case that it is nuclear). This partially solves a long-standing problem in the theory 15$]$.

Another new result in this paper is an identification of the linear dual of the representation Hopf $*$-algebra of a compact quantum group with a certain product of finite-dimensional matrix algebras. This enables us to derive a functional calculus for unbounded linear functionals on the representation algebra.

We indicate now how the paper is organized. In Section 2 we prove the existence of a Haar measure on a compact quantum semigroup in two quite different cases: when certain weak cancellation laws hold and when the quantum semigroup is abelian. In Section 3 we show the equivalence of the weak cancellation laws with Woronowicz's cancellation laws. In Section 4 we prove the result on faithfulness of the Haar measure mentioned above and finally, in Section 5 , we derive the results on the linear dual of the representation algebra already mentioned.

We refer to 9, 13 , 15] for basic results on the theory of compact quantum groups that we shall use in the paper.

\section{Existence of HaAR measure}

In this section we prove the existence and uniqueness of a Haar measure for a compact quantum semigroup satisfying certain weak cancellation laws. We begin by introducing some basic terminology and notation.

The pair $G=(A, \Delta)$ is a compact quantum semigroup if $A$ is a unital $C^{*}$-algebra and $\Delta$ is a co-multiplication of $A$; that is, $\Delta$ is a unital $*$-homomorphism from $A$ to the minimal $C^{*}$-tensor product $A \otimes A[10$, Section 6.4] such that $($ id $\otimes \Delta) \Delta=$ $(\Delta \otimes \mathrm{id}) \Delta$. We shall call $A$ the coordinate $C^{*}$-algebra of $G$ and $\Delta$ the co-multiplication of $G$.

The co-multiplication $\Delta$ induces a (dual) associative multiplication on $A^{*}$, the continuous linear dual of $A$, obtained by setting $\tau \sigma=(\tau \otimes \sigma) \Delta$. If $a \in A$ and $\tau \in A^{*}$, the elements $\tau * a$ and $a * \tau$ in $A$ are defined by setting $\tau * a=(\operatorname{id} \otimes \tau) \Delta(a)$ and $a * \tau=(\tau \otimes \mathrm{id}) \Delta(a)$. Routine computations show that, for all $\tau, \sigma \in A^{*}$ and $a \in A$, we have $(\tau \sigma)(a)=\tau(\sigma * a)=\sigma(a * \tau)$; also, $(\tau \sigma) * a=\tau *(\sigma * a)$ and $a *(\tau \sigma)=(a * \tau) * \sigma$.

If $a \in A$ and $\tau \in A^{*}$, recall that $a \tau$ and $\tau a$ in $A^{*}$ are defined by setting $(a \tau)(b)=$ $\tau(b a)$ and $(\tau a)(b)=\tau(a b)$, for all $b \in A$.

We motivate the terminology quantum "semigroup" by introducing the following class of classical examples. These examples also provide a useful testing ground for various conjectures. Let $S$ be a compact semigroup; that is, $S$ is a nonempty Hausdorff, compact space endowed with a jointly continuous, associative operation, $(s, t) \mapsto s t$. Set $A=C(S)$, the unital $C^{*}$-algebra of all complex-valued continuous functions on $S$. Identifying $C(S) \otimes C(S)$ with $C(S \times S)$ in the usual way, let $\Delta$ be the co-multiplication of $A$ defined by setting $\Delta(f)(s, t)=f(s t)$, for all $f \in A$ and $s, t \in S$. Thus, $G=(C(S), \Delta)$ is a compact quantum semigroup, which we shall say is associated to $S$. We shall also say that a compact quantum semigroup is classical if it is associated to a compact semigroup $S$ in this fashion. 
Returning to the general case, if $G=(A, \Delta)$ is a compact quantum semigroup, we shall be interested in imposing further restrictions on the nature of $A$ and $\Delta$, but even in the present generality one can obtain the following known elementary result, which is crucial for what follows. This result may be thought of as showing the "local existence of a Haar measure".

2.1. Lemma. Let $G=(A, \Delta)$ be a compact quantum semigroup. If $\tau$ is a positive linear functional of $A$, there exists a state $h$ of $A$ for which $r h=h \tau=\tau(1) h$.

Proof. Without loss of generality, we may suppose that $\tau$ is a state of $A$. Set $\tau_{n}=(1 / n) \sum_{i=1}^{n} \tau^{i}$. Then $\left(\tau_{n}\right)$ forms a sequence of states of $A$ which, by weak* compactness of the state space, has a convergent subnet with limit $h$, say. Since $\left\|\tau_{n} \tau-\tau_{n}\right\|=\left\|\tau^{n+1}-\tau\right\| / n \leq 2 / n$, the limit $h$ must satisfy the conditions $h \tau=h$ $(=\tau h)$.

Woronowicz defines a compact quantum semigroup $G=(A, \Delta)$ to be a compact quantum group if the linear spans of $(1 \otimes A) \Delta A$ and $(A \otimes 1) \Delta A$ are each dense in $A \otimes A$.

If $G$ is a classical compact quantum semigroup, associated to a compact semigroup $S$, then it is well known and easily seen that $G$ is a compact quantum group if $S$ is a compact group.

A compact quantum semigroup $G$ has the left cancellation property (of Woronowicz $)$ if the linear span of $(A \otimes 1) \Delta A$ is dense in $A \otimes A$; similarly, $G$ has the right cancellation property (of Woronowicz) if the linear span of $(1 \otimes A) \Delta A$ is dense in $A \otimes A$.

On the other hand, we shall say that $G$ has the weak left cancellation property if, whenever $\tau, \sigma \in A^{*}$ are such that $(\tau a) \sigma=0$ for all $a \in A$, we must have $\tau=0$ or $\sigma=0$. Likewise, $G$ has the weak right cancellation property if, whenever $\tau(\sigma a)=0$ for all $a \in A$, we must have $\tau=0$ or $\sigma=0$. It is clear that if $G$ has the left or right cancellation property of Woronowicz, then it has the corresponding weak cancellation property. Hence, a compact quantum group has the weak cancellation properties. We shall see in Section 3 that a compact quantum semigroup having the weak cancellation properties is a compact quantum group, but the proof of this is not easy.

In view of the important role the cancellation properties play in the theory of compact quantum groups, it is desirable to have alternative equivalent formulations. The following result provides one such formulation.

2.2. Theorem. Let $G=(A, \Delta)$ be a compact quantum semigroup. Then $G$ has the weak left cancellation property if, and only if, for every nonzero functional $\tau \in A^{*}$, the closed linear span $L_{\tau}$ in $A$ of the elements $a *(\tau b)$, where $a, b \in A$, is equal to A.

Proof. Suppose $G$ has the weak left cancellation property and $\tau$ is a nonzero functional in $A^{*}$ for which $L_{\tau} \neq A$. By the Hahn-Banach theorem, we may choose a nonzero functional $\sigma \in A^{*}$ vanishing on $L_{\tau}$. Hence, $0=\sigma(a * \tau b)=[(\tau b) \sigma](a)$, for all $a, b \in A$. It follows that $(\tau b) \sigma=0$, for all $b \in A$, and therefore, by the weak left cancellation property for $G, \tau=0$, contradicting the hypothesis. Thus, to avoid a contradiction, we must have $L_{\tau}=A$.

Suppose now conversely that $L_{\tau}=A$, whenever $\tau \neq 0$. We shall show that $G$ has the weak left cancellation property. To see this, let $\tau, \sigma \in A^{*}$ and suppose that 
$\tau \neq 0$ and that $(\tau b) \sigma=0$ for all $b \in A$. Then $\sigma(a *(\tau b))=[(\tau b) \sigma](a)=0$ for all $a \in A$. Hence, $\sigma\left(L_{\tau}\right)=0$. Since $L_{\tau}=A$, this implies that $\sigma=0$.

Of course, the corresponding right-hand version of Theorem 2.2 also holds, by a similar proof; that is, $G$ has the weak right cancellation property if, and only if, for each nonzero functional $\tau$ in $A^{*}$, the closed linear span in $A$ of the elements $(\tau a) * b$ is equal to $A$.

We now prove two results that lead to the main theorem of this section, namely, Theorem 2.5. where the existence of a Haar measure is shown for compact quantum semigroups with the weak cancellation properties.

2.3. Lemma. Let $G=(A, \Delta)$ be a compact quantum semigroup that has the weak left cancellation property. Let $\tau$ and $h$ be states of $A$ for which $\tau h=h$. Then $(\tau a) h=\tau(a) h$ for all $a \in A$. Moreover, if $\sigma$ is a positive linear functional on $A$ that is majorized by $\tau$, then $\sigma h=\sigma(1) h$.

Proof. Let $c \in A$, and set $b=h * c$. Observe that $\tau * b=b$, since $\tau * b=\tau *(h * c)=$ $(\tau h) * c=h * c=b$. Hence, writing $\hat{\tau}$ for id $\otimes \tau$, we have

$$
\begin{aligned}
& \hat{\tau}\left[(\Delta(b)-b \otimes 1)^{*}(\Delta(b)-b \otimes 1)\right] \\
& \quad=\hat{\tau}\left[\Delta\left(b^{*} b\right)-\left(b^{*} \otimes 1\right) \Delta(b)-\Delta\left(b^{*}\right)(b \otimes 1)+b^{*} b \otimes 1\right] \\
& \quad=\hat{\tau} \Delta\left(b^{*} b\right)-b^{*}(\tau * b)-(\tau * b)^{*} b+b^{*} b \\
& \quad=\hat{\tau} \Delta\left(b^{*} b\right)-b^{*} b-b^{*} b+b^{*} b=\hat{\tau} \Delta\left(b^{*} b\right)-b^{*} b .
\end{aligned}
$$

By Lemma 2.1 there is a state $k$ of $A$ for which $k \tau=k$. Applying $k$ to these equations we get $(k \otimes \tau)\left[(\Delta(b)-b \otimes 1)^{*}(\Delta(b)-b \otimes 1)\right]=(k \otimes \tau) \Delta\left(b^{*} b\right)-k\left(b^{*} b\right)=$ $k \tau\left(b^{*} b\right)-k\left(b^{*} b\right)=0$, since $k \tau=k$. Hence, by the Cauchy-Schwarz inequality applied to the positive state $k \otimes \tau$, we have $(k \otimes \tau)[(d \otimes a)(\Delta(b)-b \otimes 1)]=0$, for all $a, d \in A$. Consequently, $(k d \otimes \tau a) \Delta(b)=\tau(a)(k d)(b)$. Rephrasing in terms of $c$, we get $[(k d)(\tau a) h](c)=\tau(a)[(k d) h](c)$. Since $c$ is an arbitrary element in $A$, we therefore have $(k d)((\tau a) h-\tau(a) h)=0$ for all $d \in A$ and consequently, by weak left cancellation in $G,(\tau a) h=\tau(a) h$, as required.

Now suppose that $\sigma$ is a positive linear functional on $A$ majorized by $\tau$. Since

$$
(k \otimes \tau)\left[(\Delta(b)-b \otimes 1)^{*}(\Delta(b)-b \otimes 1)\right]=0,
$$

we have

$$
(k \otimes \sigma)\left[(\Delta(b)-b \otimes 1)^{*}(\Delta(b)-b \otimes 1)\right]=0 .
$$

Hence, by the Cauchy-Schwarz inequality, $(k \otimes \sigma)[(d \otimes a)(\Delta(b)-b \otimes 1)]=0$, for all $a, d \in A$. Arguing as before, with $a=1$ and with $\sigma$ in place of $\tau$, we get $\sigma h=\sigma(1) h$, as required.

2.4. Theorem. Let $G=(A, \Delta)$ be a compact quantum semigroup that has the weak left cancellation property. Then there exists a state $h$ of $A$ for which $\tau h=\tau(1) h$ for all $\tau \in A^{*}$.

Proof. For each positive $\tau \in A^{*}$, let $K_{\tau}$ denote the set of states $h$ of $A$ for which $\tau h=\tau(1) h$. It is clear that $K_{\tau}$ is weak ${ }^{*}$ closed in $A^{*}$ and, by Lemma 2.1. $K_{\tau} \neq \emptyset$. If $\sigma$ is a positive linear functional for which $\tau \leq \sigma$, then $K_{\sigma} \subseteq K_{\tau}$, by Lemma 2.3 Hence, for all positive linear functionals $\tau$ and $\sigma$, we have $K_{\tau+\sigma} \subseteq K_{\tau} \cap K_{\sigma}$. It follows that the family of sets $\left(K_{\tau}\right)_{\tau}$ has finite-intersection property and therefore, by weak* compactness of the state space of $A$, there is a state $h$ belonging to the intersection $\bigcap_{\tau} K_{\tau}$. Hence, $\tau h=\tau(1) h$, for $\tau$ any positive linear functional, 
and therefore for $\tau$ any bounded linear functional, since positive linear functionals linearly $\operatorname{span} A^{*}$.

The state $h$ in Theorem 2.4 is not necessarily unique. For instance, take $A$ to be any unital $C^{*}$-algebra of dimension greater than one, and let $\Delta$ be the comultiplication on $A$ defined by setting $\Delta(a)=1 \otimes a$. It is clear that the compact quantum semigroup $G=(A, \Delta)$ has the weak left cancellation property, since $\tau \sigma=\tau(1) \sigma$, for all $\tau, \sigma \in A^{*}$. Equally obvious, every state of $A$ has the "absorbing property" of $h$ in Theorem 2.4 .

Now suppose that $G=(A, \Delta)$ is a compact quantum semigroup and that $\theta$ is the flip automorphism of $A \otimes A$; that is, $\theta$ is the unique $*$-homomorphism of $A \otimes A$ into itself determined by the condition $\theta(a \otimes b)=b \otimes a$, for all $a, b \in A$. Then it is easily seen that $G^{\text {op }}=(A, \theta \Delta)$ is a compact quantum semigroup, called the opposite compact quantum semigroup of $G$. Observe that the dual multiplication associated to $G^{\text {op }}$ is the opposite of the dual multiplication associated to $G$.

The following is the principal result of this section. Its proof is an easy consequence of Theorem 2.4

2.5. Theorem. Let $G=(A, \Delta)$ be a compact quantum semigroup that has the weak left and right cancellation properties. Then there is a unique state $h$ of $A$ for which $\tau h=h \tau=\tau(1) h$, for all $\tau \in A^{*}$.

Proof. It is clear that the hypothesis of Theorem 2.4 applies both for $G$ and $G^{\text {op }}$, since $G$ has the weak left and right cancellation properties. Hence, by Theorem [2.4, there exist states $h$ and $h^{\prime}$ of $A$ for which $\tau h=\tau(1) h$ and $h^{\prime} \tau=\tau(1) h^{\prime}$ for all $\tau \in A^{*}$. Consequently, $h^{\prime} h=h^{\prime}(1) h=h$ and $h^{\prime} h=h(1) h^{\prime}=h^{\prime}$. Therefore, $h=h^{\prime}$. This proves the result.

2.6. Corollary (S. L. Woronowicz [15]). Let $G$ be a compact quantum group. Then there is a unique state $h$ of $A$ for which $\tau h=h \tau=\tau(1) h$ for all $\tau \in A^{*}$.

If a compact quantum semigroup $G=(A, \Delta)$ admits a (necessarily unique) state $h$ of $A$ for which $\tau h=h \tau=\tau(1) h$, for all $\tau \in A^{*}$, then $h$ is called the Haar measure of $G$. Observe that if $a \in A$ and $\tau \in A^{*}$, then $\tau(h * a)=(\tau h)(a)=\tau(1) h(a)$. Hence, $\tau(h * a-h(a) 1)=0$. Since $\tau$ is arbitrary, this implies that $h * a=h(a) 1$. Similar reasoning shows that $a * h=h(a) 1$.

Conversely, if $h$ is a state of $A$ for which $h * a=a * h=h(a) 1$, for all $a \in A$, then $h$ is the Haar measure of $G$, as is easily verified.

Of course, the content of Theorem 2.5 is the assertion that a compact quantum semigroup having the weak left and right cancellation properties has a unique Haar measure.

The following known result is useful for identifying the Haar measure. The proof we give, using Lemma 2.3, is new.

2.7. Theorem. Let $G=(A, \Delta)$ be a compact quantum semigroup that has the weak left and right cancellation properties. Suppose that $\tau$ and $h$ are states of $A$, that $\tau$ is faithful and that $\tau h=h$. Then $h$ is the Haar measure of $G$.

Proof. By Lemma 2.3 $(\tau a) h=\tau(a) h$ for all $a \in A$. Therefore, if $b \in A$, we have $(\tau a)(h * b-h(b) 1)=((\tau a) h)(b)-\tau(a) h(b)=0$. Hence, $h * b-h(b) 1$ belongs to the left kernel of $\tau$. Since $\tau$ is faithful, this implies that $h * b-h(b) 1=0$. Now let $h_{0}$ be the Haar measure of $G$. Then $h_{0}(b)-h(b)=\left(h_{0} h\right)(b)-h(b)=h_{0}(h * b-h(b) 1)=0$. Therefore, $h_{0}=h$. 
A compact quantum semigroup $G=(A, \Delta)$ is said to be abelian if $G=G^{\mathrm{op}}$. We introduce abelian compact quantum semigroups at this point since we are going to show in the following theorem that they always admit a Haar measure, even in the absence of any cancellation-property assumption. The result, and the method of proof, appear to be new in this context.

2.8. Theorem. Let $G=(A, \Delta)$ be an abelian compact quantum semigroup. Then $G$ admits a unique Haar measure.

Proof. Since $\sigma \tau=\tau \sigma$ and the map, $\tau \mapsto \sigma \tau$, is weak ${ }^{*}$ continuous for each element $\sigma \in S$, the state space $S$ of $A$ is a commutative compact topological semigroup in the sense of [2], when endowed with the induced multiplication and the relative weak* topology. Hence, the kernel of $S$, that is, the smallest nonempty subset $K$ of $S$ for which $S K \subset K$, is a group under the induced multiplication [2, Corollary 2.10]. Since $S$ is convex, [2, Theorem 3.3] implies that $K$ consists of idempotents. Hence, $K$ is a singleton, $\{h\}$ say. Therefore, $h$ is a state of $A$ for which $\tau h=h \tau=h$, for all states $\tau$ of $A$. Since the states linearly span $A^{*}$, we must have $\tau h=h \tau=\tau(1) h$, for all $\tau \in A^{*}$.

We are now in a position to show that existence of a unique Haar measure on a compact quantum semigroup $G=(A, \Delta)$ does not imply that $G$ has the weak left or right cancellation properties. To see this, consider the compact semigroup $\mathbf{D}$, the closed unit disc in the complex plane with the usual multiplication, and let $G=(C(\mathbf{D}), \Delta)$ be the associated compact quantum semigroup. We shall show that $G$ has a unique Haar measure, but that the weak cancellation properties do not hold. Clearly, $G$ is abelian and therefore, by Theorem 2.8, it admits a unique Haar measure. In fact, an elementary computation shows that $\delta_{0}$, the functional on $C(\mathbf{D})$ obtained by evaluation at the origin, is the Haar measure of $G$. Moreover, if $f, g \in C(\mathbf{D})$, then another simple computation shows that $f *\left(\delta_{0} g\right)=f(0) g(0) 1$. Hence, the closed linear span in $C(\mathbf{D})$ of all such elements $f *\left(\delta_{0} g\right)$ is $\mathbf{C} 1$. Therefore, by Theorem 2.2, $G$ does not have the weak (left) cancellation property.

A natural question now arises: Is it the case that every compact quantum semigroup admits a Haar measure? The answer is no. To see this, let $S$ be the compact semigroup consisting of the set of triples $(x, y, z)$, where $x, y, z \in[0,1]$, and the multiplication is defined by setting $(x, y, z)\left(x^{\prime}, y^{\prime}, z^{\prime}\right)=\left(x, y^{\prime}, x y^{\prime}\right)$. Let $G=(C(S), \Delta)$ be the associated compact quantum semigroup. We claim that $G$ admits no Haar measure. Suppose otherwise, and let $h$ be a Haar measure. For $s \in S$, let $\delta_{s}$ be the element of $C(S)^{*}$ given by evaluation at $s$. Let $e=(0,0,0)$. Since the elements $\delta_{s}(s \in S)$ form the set of pure states of $C(S)$, and therefore their closed convex hull in the weak* topology is the state space of $C(S)$, the element $h=\delta_{e} h \delta_{e}$ belongs to the weak* closed convex hull of the elements $\delta_{e} \delta_{s} \delta_{e}(s \in S)$. However, $\delta_{e} \delta_{s} \delta_{e}=\delta_{e s e}=\delta_{e}$. Hence, $h=\delta_{e}$. This implies that $\delta_{(0,1,0)}=\delta_{e} \delta_{(0,1,0)}=h \delta_{(0,1,0)}=h=\delta_{e}$. Therefore, $(0,1,0)=e=(0,0,0)$. Of course, this is impossible, so - as claimed $-G=(C(S), \Delta)$ admits no Haar measure.

\section{The equivalence of the Weak and Woronowicz CANCELlation laWs}

We begin this section by recalling some well-known facts. Let $B$ be a $C^{*}$-algebra and denote by $M(B)$ the multiplier algebra of $B$. Let $\omega \in B^{*}$ be strictly continuous (that is, continuous with respect to the strict topology on $B$ ). Then $\omega$ has a strictly 
continuous linear extension $\bar{\omega}$ to $M(B)$ [10, Theorem A.11]. Since $B$ is dense in $M(B)$ relative to the strict topology, this extension is unique. One can easily check that $\bar{\omega}$ is bounded and $\|\bar{\omega}\|=\|\omega\|$. Henceforth, we denote $\bar{\omega}$ by the same symbol $\omega$.

Now suppose that $C$ is another $C^{*}$-algebra. If $\omega \in B^{*}$, it is well known that there is a unique bounded, linear map $\omega \otimes$ id from $B \otimes C$ to $C$ such that $(\omega \otimes \mathrm{id})(b \otimes c)=$ $\omega(b) c$, for all $b \in B$ and $c \in C$. Moreover, $\| \omega \otimes$ id $\|=\| \omega \|$. The map $\omega \otimes$ id is called a slice map 8 , 12. It admits a unique bounded, linear extension from $M(B \otimes C)$ to $M(C)$, also denoted by $\omega \otimes \mathrm{id}$, that is strictly continuous on the unit ball of $M(B \otimes C)$; see [4], for example.

We shall use these results in the case that $B=K(H)$, the $C^{*}$-algebra of compact operators on a Hilbert space $H$. Of course, $M(K(H))=B(H)$, as is well known. If $x, y \in H$, denote by $\omega_{x, y}$, the functional $T \mapsto\langle T(x), y\rangle$ on $B(H)$. We shall also use the notation $\omega_{x, y}$ for restrictions to various subalgebras of $B(H)$. The context, or an explicit statement, will make clear what is the domain of $\omega_{x, y}$. Regarded as a functional on $K(H), \omega_{x, y}$ is strictly continuous.

3.1. Theorem. Let $B=K(H)$ where $H$ is a Hilbert space, and let $\omega$ be the restriction of $\omega_{x, y}$ to $K(H)$, where $x, y \in H$. Let $C$ be a unital $C^{*}$-algebra. Then $\omega \otimes \mathrm{id}$ is continuous for the strict topology on $M(B \otimes C)$ and the norm topology on $C$.

Proof. Let $\left(U_{n}\right)$ be a net in $M(B \otimes C)$ converging in the strict topology to an element $U$. Let $P \in B$ be the orthogonal projection of $H$ onto $\mathbf{C} y$. Obviously, $\omega P=\omega$ and therefore $(\omega \otimes$ id $)((P \otimes 1) V)=(\omega \otimes$ id $)(V)$, for all $V \in M(B \otimes C)$. Since the net $(P \otimes 1) U_{n}$ converges to $(P \otimes 1) U$ in norm, the net $(\omega \otimes$ id $)\left((P \otimes 1) U_{n}\right)$ converges to $(\omega \otimes$ id $)((P \otimes 1) U)$ in norm; that is, $(\omega \otimes$ id $)\left(U_{n}\right)$ converges to $(\omega \otimes$ id $)(U)$ in norm.

We now show that the weak cancellation properties are equivalent to Woronowicz's cancellation properties. We originally gave a more indirect proof of this result, in which we assumed faithfulness of the Haar measure $h$. We thank our colleague Alfons Van Daele who showed us the following, more direct, proof that does not require faithfulness of $h$.

3.2. Theorem. Let $G=(A, \Delta)$ be a compact quantum semigroup. Then $G$ is a compact quantum group if, and only if, $G$ has the weak left and right cancellation properties.

Proof. We have already seen that a compact quantum group has the weak cancellation properties. Assume conversely that $G$ has the weak cancellation properties. We shall show that $G$ must then have the Woronowicz cancellation properties. In fact, we shall show that $G$ has the Woronowicz left cancellation property. It will then follow, since $G^{\text {op }}$ also has the weak cancellation properties, that $G^{\text {op }}$ has the Woronowicz left cancellation property and therefore $G$ has the Woronowicz right cancellation property.

Let $\varphi: A \rightarrow B(H)$ be the GNS representation associated to the Haar measure $h$, and let $z$ be the canonical cyclic vector in $H$. Then $h(a)=\omega_{z, z} \varphi(a)$ for all $a \in A$. Set $C=A \otimes A$.

Choose an orthonormal basis $\left(e_{i}\right)_{i \in I}$ for $H$, and let $P_{i}$ be the orthogonal projection of $H$ onto $\mathbf{C} e_{i}$. Then $\sum_{i}\left(P_{i} \otimes 1\right)=1$ in the strict topology of $M(K(H) \otimes C)$. 
Hence, for all $U, V$ belonging to this multiplier algebra we have $\sum_{i} U\left(P_{i} \otimes 1\right) V=$ $U V$, again in the strict topology. Therefore, by Theorem 3.1

$$
\sum_{i}\left(\omega_{z, z} \otimes \mathrm{id}\right)\left(U\left(P_{i} \otimes 1\right) V\right)=\left(\omega_{z, z} \otimes \mathrm{id}\right)(U V)
$$

in the norm topology in $C$. One can readily verify that

$$
\left(\omega_{z, z} \otimes \mathrm{id}\right)\left(U\left(P_{i} \otimes 1\right) V\right)=\left(\omega_{e_{i}, z} \otimes \mathrm{id}\right)(U)\left(\omega_{z, e_{i}} \otimes \mathrm{id}\right)(V),
$$

by first checking the formula in the case that $U, V \in K(H) \otimes_{\text {alg }} C$ and then extending by continuity, using Theorem 3.1. Hence, we have, in the norm topology of $C$,

$$
\sum_{i}\left(\omega_{e_{i}, z} \otimes \mathrm{id}\right)(U)\left(\omega_{z, e_{i}} \otimes \mathrm{id}\right)(V)=\left(\omega_{z, z} \otimes \mathrm{id}\right)(U V) .
$$

Since $K(H) \otimes C$ is an essential ideal in $B(H) \otimes C$, we may, and do, embed $B(H) \otimes$ $C$ as a unital $C^{*}$-subalgebra of $M(K(H) \otimes C)$. Hence, $\varphi(A) \otimes C \subseteq M(K(H) \otimes C)$. It follows that we may apply Equation (11) with $U, V \in \varphi(A) \otimes C$. Let $\omega_{e_{i}, z}^{\prime}=\omega_{e_{i}, z} \varphi$ and $\omega_{z, e_{i}}^{\prime}=\omega_{z, e_{i}} \varphi$. Then, since $h=\omega_{z, z} \varphi$, we have, in the norm topology of $C$,

$$
\sum_{i}\left(\omega_{e_{i}, z}^{\prime} \otimes \mathrm{id}\right)(U)\left(\omega_{z, e_{i}}^{\prime} \otimes \mathrm{id}\right)(V)=(h \otimes \mathrm{id})(U V),
$$

for all $U, V \in A \otimes A \otimes A$.

Now take $U=\Delta(b) \otimes 1$ and $V=(\Delta \otimes$ id $) \Delta(a)$, where $a, b \in A$. Let $h^{\prime}: A \rightarrow A$ be defined by $h^{\prime}(a)=h(a) 1_{A}$. Then

$$
\begin{aligned}
(h \otimes \mathrm{id})(U V) & =(h \otimes \mathrm{id})(\Delta \otimes \mathrm{id})((b \otimes 1) \Delta(a))=\left(h^{\prime} \otimes \mathrm{id}\right)((b \otimes 1) \Delta(a)) \\
& =1 \otimes(h \otimes \mathrm{id})((b \otimes 1) \Delta(a))=1 \otimes a *(h b) .
\end{aligned}
$$

Hence, by Equation (2),

$$
1 \otimes a *(h b)=\sum_{i} c_{i},
$$

where $c_{i}=\left(\omega_{e_{i}, z}^{\prime} \otimes \mathrm{id}\right)(U)\left(\omega_{z, e_{i}}^{\prime} \otimes \mathrm{id}\right)(V)$. We claim now that $1 \otimes a *(h b)$ belongs to the closed linear span of $(A \otimes 1) \Delta(A)$. To see this we need only show that each element $c_{i}$ belongs to $(A \otimes 1) \Delta(A)$. Clearly, $\left(\omega_{e_{i}, z}^{\prime} \otimes \mathrm{id}\right)(U)=\left(\left(\omega_{e_{i}, z}^{\prime} \otimes \mathrm{id}\right) \Delta(b)\right) \otimes 1$ and this obviously belongs to $A \otimes 1$. Also,

$$
\begin{aligned}
\left(\omega_{z, e_{i}}^{\prime} \otimes \mathrm{id}\right)(V) & =\left(\omega_{z, e_{i}}^{\prime} \otimes \mathrm{id}\right)(\Delta \otimes \mathrm{id}) \Delta(a) \\
& =\left(\omega_{z, e_{i}}^{\prime} \otimes \mathrm{id}\right)(\mathrm{id} \otimes \Delta) \Delta(a)=\Delta\left(\omega_{z, e_{i}}^{\prime} \otimes \mathrm{id}\right) \Delta(a) .
\end{aligned}
$$

Here we have used the easily verified equation $(\omega \otimes$ id $)(\mathrm{id} \otimes \Delta)(c)=\Delta(\omega \otimes$ id $)(c)$, which is true for all $c \in A \otimes A$ and all $\omega \in A^{*}$. Hence, $\left(\omega_{z, e_{i}}^{\prime} \otimes \mathrm{id}\right)(V)$ belongs to $\Delta(A)$. Thus, we have shown that $c_{i} \in(A \otimes 1) \Delta(A)$, for all indices $i$. Therefore, $1 \otimes a *(h b)$ belongs to the closed linear span of $(A \otimes 1) \Delta(A)$, as claimed. Since the elements of the form $a *(h b)$ have closed linear span equal to $A$, by Theorem 2.2, it follows that $G$ satisfies Woronowicz's left cancellation law. This proves the theorem. 


\section{FAithfulness of HAar MEASURE}

In this section we give some conditions implying faithfulness of the Haar measure of a compact quantum group. Our most important result here is Theorem 4.1, which solves in great generality a long-standing problem in the theory; namely, to give an algebraic condition, not referring to the Haar measure, that is equivalent to its faithfulness 15 .

In the classical theory, the Haar measure (integral) $h$ is automatically faithful. It is a defect of the quantum theory that this is no longer the case. (Consider $A=C^{*}\left(\mathbf{F}_{2}\right)$, where $\mathbf{F}_{2}$ is the free group on two generators. In this case, the Haar integral is the canonical trace, and this is not faithful.) The remedy, of course, is to find a suitable extra axiom that a compact quantum group should satisfy to ensure faithfulness of $h$ (but that does not require a priori existence of $h$ ). In the opinion of the authors, the following theorem provides the required suitable extra axiom, and we conjecture that the exactness condition imposed in the hypothesis of the theorem is unnecessary.

4.1. Theorem. Suppose that the coordinate $C^{*}$-algebra $A$ of the compact quantum group $(A, \Delta)$ is exact. Then the following conditions are equivalent:

(1) The Haar measure $h$ of $(A, \Delta)$ is faithful.

(2) A closed ideal $I$ of $A$ that satisfies the invariance condition $\Delta(I) \subseteq I \otimes I$ must be trivial; that is, $I=0$ or $I=A$.

Proof. Suppose first that Condition (1) holds, and let $I$ be a closed ideal of $A$ for which $\Delta(I) \subseteq I \otimes I$. If $I \neq A$, then there exists a unital functional $\tau \in A^{*}$ that vanishes on $\bar{I}$. If $a$ belongs to $I$ and $b \in A$, we have $\Delta(b a) \in I \otimes I$. Since $\tau \otimes h$ vanishes on $I \otimes I$, we have $h(b a)=\tau(1) h(b a)=(\tau h)(b a)=(\tau \otimes h) \Delta(b a)=0$. Therefore, $a$ belongs to the left kernel of $h$. By faithfulness of $h, a=0$. Hence, $I=0$. Thus, Condition (1) implies (2) (even without assuming $A$ is exact).

Now suppose conversely that Condition (2) holds. Let $I$ be the left kernel of $h$. Then $I$ is a closed two-sided ideal of $A$ [9 Proposition 7.9]. If $\tau$ is a positive linear functional on $A$ and $a \in I$, then $(\tau \otimes h)\left(\Delta\left(a^{*}\right) \Delta(a)\right)=(\tau h)\left(a^{*} a\right)=\tau(1) h\left(a^{*} a\right)=0$. Hence, since $\tau \otimes h$ is positive, $(\tau \otimes h)(c \Delta(a))=0$ for all $c \in A \otimes A$. Therefore, (id $\otimes h)(c \Delta(a))=0$, since $\tau$ was an arbitrary positive linear functional on $A$. Now take $c=1 \otimes b$, where $b \in A$ and let $\sigma \in A^{*}$. Then $(h b)(a * \sigma)=(\sigma(h b))(a)=$ $(\sigma \otimes h b) \Delta(a)=\sigma(\mathrm{id} \otimes h)((1 \otimes b) \Delta(a))=0$. Therefore, $a * \sigma \in I$. Thus, we have shown that $I * \sigma \subseteq I$, for all $\sigma \in A^{*}$. Likewise, one can show that $\sigma * I \subseteq I$.

We claim now that $\Delta(I) \subseteq A \otimes I$. Otherwise, there exists $a \in I$ such that $\Delta(a)$ does not belong to $A \otimes I$. Let $\pi$ be the quotient map from $A$ to $A / I$. Since $A$ is an exact $C^{*}$-algebra, the kernel of the homomorphism id $\otimes \pi: A \otimes A \rightarrow A \otimes(A / I)$ is $A \otimes$ ker $\pi=A \otimes I$. Hence, $(\mathrm{id} \otimes \pi) \Delta(a) \neq 0$. It follows that there exist bounded linear functionals $\sigma$ and $\tau^{\prime}$ on $A$ and $A / I$, respectively, such that $\left(\sigma \otimes \tau^{\prime}\right)($ id $\otimes \pi) \Delta(a) \neq 0$. That is, $(\sigma \otimes \tau) \Delta(a) \neq 0$, where $\tau=\tau^{\prime} \pi \in A^{*}$. However, $\tau(I)=0$ and $a * \sigma \in I$, since $I * \sigma \subseteq I$. So $(\sigma \otimes \tau) \Delta(a)=(\sigma \tau)(a)=\tau(a * \sigma)=0$, a contradiction. It follows that $\Delta(I)$ is contained in $A \otimes I$. A similar argument, using the inclusion $\sigma * I \subseteq I$ for $\sigma \in A^{*}$, shows that $\Delta(I)$ is contained in $I \otimes A$. Now, since $\Delta$ is a homomorphism and $I$ is the linear span of $I^{2}$, it follows easily that $\Delta(I) \subseteq I \otimes I$. It now follows from Condition (2) that $I=0$. Hence, $h$ is faithful. Thus, we have shown that Condition (2) implies (1). 
The next result is an immediate consequence of Theorem [2.7 We highlight it here since it gives a nice condition guaranteeing that the Haar measure is faithful, and since our following result, Theorem 4.3 is a consequence of it.

4.2. Theorem. Suppose that $(A, \Delta)$ is a compact quantum group and that $A$ admits a faithful state $\tau$ for which $\tau^{2}=\tau$. Then $\tau$ is the Haar measure of $(A, \Delta)$.

Example. Let $\Gamma$ be a discrete group, and let $L: x \mapsto L_{x}$ be the left regular representation of $\Gamma$ on $\ell^{2}(\Gamma)$. Let $A=C_{\mathrm{r}}^{*}(\Gamma)$ be the reduced group $C^{*}$-algebra of $\Gamma$; that is, $A$ is the $C^{*}$-subalgebra of $B\left(\ell^{2}(\Gamma)\right)$ generated by the operators $L_{x}$ $(x \in \Gamma)$. The linear map $\Delta$ defined on $A$ by $\Delta\left(L_{x}\right)=L_{x} \otimes L_{x}$, for all $x \in \Gamma$, is a co-multiplication of $A$. It is well known that $G=(A, \Delta)$ is a compact quantum group. It is also well known that $C_{\mathrm{r}}^{*}(\Gamma)$ admits a faithful tracial state tr given by $\operatorname{tr}\left(L_{x}\right)=0$, if $x$ is an element of $\Gamma$ that is not equal to the unit of $\Gamma$. Clearly, $\operatorname{tr}^{2}=\operatorname{tr}$. Hence, by Theorem 4.2, $\operatorname{tr}$ is the Haar measure of $G$.

4.3. Theorem. Suppose that $(A, \Delta)$ is a compact quantum group and that $A$ admits a unique tracial state $\tau$. Suppose that $\tau$ is faithful. Then $\tau$ is the Haar measure of $(A, \Delta)$.

Proof. Since the product in $A^{*}$ of two tracial states is clearly a tracial state, and since there is only one tracial state, we have $\tau^{2}=\tau$. The result now follows from Theorem 4.2 .

In Theorem 4.3 we can replace the hypothesis on $\tau$ by assuming only that $A$ admits a unique faithful tracial state $\tau$. For then $\tau$ is, in fact, the unique tracial state of $A$. For, if $\sigma$ is another tracial state of $A$, then $(\tau+\sigma) / 2$ is a faithful tracial state, and therefore $(\tau+\sigma) / 2=\tau$, from which $\sigma=\tau$.

It is an interesting question whether a compact quantum semigroup admitting a faithful Haar measure is necessarily a compact quantum group. We conjecture that the answer is affirmative. Our next result is evidence that supports this conjecture.

A compact quantum semigroup is said to be finite if its coordinate $C^{*}$-algebra is finite dimensional.

4.4. Theorem. Let $(A, \Delta)$ be a compact quantum semigroup admitting a faithful Haar measure $h$. If $(A, \Delta)$ is finite or classical, it is a compact quantum group.

Proof. Let $\alpha_{l}$ and $\alpha_{r}$ be the unique linear operators from $A \otimes_{\text {alg }} A$ to $A \otimes A$ for which $\alpha_{l}(a \otimes b)=\Delta(b)(a \otimes 1)$ and $\alpha_{r}(a \otimes b)=\Delta(a)(1 \otimes b)$, for all $a, b \in A$. Let $a_{1}, \ldots, a_{n}$ and $b_{1}, \ldots, b_{n}$ belong to $A$, and let $c=\sum_{i} a_{i} \otimes b_{i}$. Here, and in the rest of this proof, all summations are over the range $1, \ldots, n$. We have

$$
\begin{aligned}
(h \otimes & h)\left(\left(\alpha_{r}(c)\right)^{*} \alpha_{r}(c)\right)=\sum_{i, j}(h \otimes h)\left(\left(1 \otimes b_{i}^{*}\right) \Delta\left(a_{i}^{*} a_{j}\right)\left(1 \otimes b_{j}\right)\right) \\
= & \sum_{i, j} h\left(b_{i}^{*}(h \otimes \mathrm{id})\left(\Delta\left(a_{i}^{*} a_{j}\right)\right) b_{j}\right)=\sum_{i, j} h\left(b_{i}^{*} h\left(a_{i}^{*} a_{j}\right) b_{j}\right) \\
= & \sum_{i, j} h\left(a_{i}^{*} a_{j}\right) h\left(b_{i}^{*} b_{j}\right)=(h \otimes h)\left(c^{*} c\right) .
\end{aligned}
$$

Hence, if $\alpha_{r}(c)=0$, then $(h \otimes h)\left(c^{*} c\right)=0$. Since $h$ is faithful, so is $h \otimes h$ [12, p. 3]. Hence, $c=0$. Therefore, $\alpha_{r}$ is injective. A similar argument shows that $a_{l}$ is injective. 
Suppose first that $A$ is finite dimensional. Then $A \otimes_{\text {alg }} A$ is also finite dimensional and equal to $A \otimes A$. Hence, the injective linear operators $\alpha_{l}$ and $\alpha_{r}$ are bijections. It follows immediately that $(A, \Delta)$ satisfies the cancellation laws, and therefore $(A, \Delta)$ is a compact quantum group in this case.

Now suppose that $(A, \Delta)$ is classical, and let $S$ be the associated compact semigroup, so that $A=C(S)$ and $A \otimes A=C(S \times S)$ and $\Delta(f)(s, t)=f(s t)$, for all $f \in A$ and $s, t \in S$. In this case the maps $\alpha_{l}$ and $\alpha_{r}$ are the restrictions of the *-homomorphisms $\hat{\alpha}_{l}$ and $\hat{\alpha}_{r}$ from $A \otimes A$ to itself induced by the continuous maps $\theta_{l}, \theta_{r}: S \times S \rightarrow S \times S$ defined by $\theta_{l}(s, t)=(s, s t)$ and $\theta_{r}(s, t)=(s t, t)$. The equality $(h \otimes h)\left(\alpha_{r}(c)^{*} \alpha_{r}(c)\right)=(h \otimes h)\left(c^{*} c\right)$, for $c \in A \otimes_{\text {alg }} A$ clearly extends to the similar equality $(h \otimes h)\left(\hat{\alpha}_{r}(c)^{*} \hat{\alpha}_{r}(c)\right)=(h \otimes h)\left(c^{*} c\right)$, for $c \in A \otimes A$. Hence, arguing as before, $\hat{\alpha}_{r}$ is injective. A corresponding argument for $\hat{\alpha}_{l}$ shows that it also is injective. Hence, $\theta_{l}$ and $\theta_{r}$ are surjective. It follows that $s S=S s=S$, for all $s \in S$ and therefore that $S$ is algebraically a group. Hence, $\theta_{l}$ and $\theta_{r}$ are injective and consequently homeomorphisms. This implies that $\hat{\alpha}_{l}$ and $\hat{\alpha}_{r}$ are automorphisms. Therefore, the linear spans of $\Delta(A)(A \otimes 1)$ and $\Delta(A)(1 \otimes A)$ are each dense in $A \otimes A$. Hence, $(A, \Delta)$ is a compact quantum group in this case also.

This result should be compared with [3, Theorem 4.2]. There it is shown that if $(A, \Delta)$ is a compact quantum semigroup having a faithful Haar measure and a bounded co-unit, it is necessarily a compact quantum group.

\section{A representation of $\mathcal{A}^{\prime}$}

In this section we identify the linear dual space of the representation algebra of a compact quantum group as a product of finite matrix algebras. We then use this to construct a functional calculus.

Let $(A, \Delta)$ be a compact quantum group, and let $\mathcal{A}$ be its unique dense Hopf *-subalgebra (its representation algebra). Denote by $\mathcal{A}^{\prime}$ the linear space of all linear functionals on $\mathcal{A}$. If $\tau$ and $\sigma$ belong to $\mathcal{A}^{\prime}$, define the functionals $\tau \sigma$ and $\tau^{*}$, both belonging to $\mathcal{A}^{\prime}$, by setting $(\tau \sigma)(a)=(\tau \otimes \sigma) \Delta(a)$ and $\tau^{*}(a)=\tau\left(\kappa(a)^{*}\right)^{-}$for all $a \in \mathcal{A}$. Here $\kappa$ is the antipode of $(\mathcal{A}, \Delta)$. It is well known that $\mathcal{A}^{\prime}$ becomes a unital *-algebra when endowed with the (dual) multiplication, $(\tau, \sigma) \mapsto \tau \sigma$, and the (dual) involution, $\tau \mapsto \tau^{*}$.

Denote by $\widehat{G}$ the set of equivalence classes of irreducible unitary co-representations of $G=(A, \Delta)$. If $\alpha \in \widehat{G}$, choose a unitary co-representation $U^{\alpha}$ belonging to $\alpha$. Let $U_{i j}^{\alpha}$ be the matrix entries of $U^{\alpha}$ relative to some basis of the Hilbert space underlying $U^{\alpha}$. Let $N_{\alpha}$ be the dimension of this Hilbert space. If $\tau \in \mathcal{A}^{\prime}$, denote by $\pi_{\alpha}(\tau)$ the matrix in $M_{N_{\alpha}}(\mathbf{C})$ whose $i j$-th entry is $\tau\left(U_{i j}^{\alpha}\right)$. Let $\pi(\tau)$ be the element $\left(\pi_{\alpha}(\tau)\right)_{\alpha}$ of the direct product algebra $\prod_{\alpha} M_{N_{\alpha}}(\mathbf{C})$. This gives a map $\pi$ from $\mathcal{A}^{\prime}$ to $\prod_{\alpha} M_{N_{\alpha}}(\mathbf{C})$ that is clearly linear.

We endow $\mathcal{A}^{\prime}$ with the topology of pointwise convergence and $\prod_{\alpha} M_{N_{\alpha}}(\mathbf{C})$ with the product topology.

5.1. Theorem. The map $\pi$ is a homeomorphic *-isomorphism of $\mathcal{A}^{\prime}$ onto $\prod_{\alpha} M_{N_{\alpha}}(\mathbf{C})$.

Proof. First, we show multiplicativity of $\pi$. If $\tau, \sigma \in \mathcal{A}^{\prime}$, then $\pi_{\alpha}(\tau \sigma)_{i j}=\tau \sigma\left(U_{i j}^{\alpha}\right)$ $=(\tau \otimes \sigma) \Delta\left(U_{i j}^{\alpha}\right)=\sum_{r=1}^{N_{\alpha}} \tau\left(U_{i r}^{\alpha}\right) \sigma\left(U_{r j}^{\alpha}\right)=\sum_{r=1}^{N_{\alpha}} \pi_{\alpha}(\tau)_{i r} \pi_{\alpha}(\sigma)_{r j}$. Therefore, $\pi_{\alpha}(\tau \sigma)$ $=\pi_{\alpha}(\tau) \pi_{\alpha}(\sigma)$ and $\pi(\tau \sigma)=\pi(\tau) \pi(\sigma)$, as required. 
Next, we show $\pi$ is $*$-preserving. If $\tau \in \mathcal{A}^{\prime}, \pi_{\alpha}\left(\tau^{*}\right)_{i j}=\tau^{*}\left(U_{i j}^{\alpha}\right)=\left(\tau\left(\left(\kappa\left(U_{i j}^{\alpha}\right)\right)^{*}\right)\right)^{-}$ $=\left(\tau\left(\left(U_{j i}^{\alpha *}\right)^{*}\right)\right)^{-}=\left(\tau\left(U_{j i}^{\alpha}\right)\right)^{-}=\left(\pi_{\alpha}(\tau)^{*}\right)_{i j}$. Hence, $\pi_{\alpha}\left(\tau^{*}\right)=\pi_{\alpha}(\tau)^{*}$, for all $\alpha \in \widehat{G}$, and therefore $\pi\left(\tau^{*}\right)=\pi(\tau)^{*}$.

Injectivity of $\pi$ is an obvious consequence of the fact that the elements $U_{i j}^{\alpha}$ linearly span $\mathcal{A}$, and surjectivity of $\pi$ is an equally obvious consequence of the fact that these elements are also linearly independent.

To see that $\pi$ is continuous, let $\left(\tau_{n}\right)$ be a net in $\mathcal{A}^{\prime}$ converging to $\tau$. Then the net $\left(\tau_{n}\left(U_{i j}^{\alpha}\right)\right)$ converges to $\tau\left(U_{i j}^{\alpha}\right)$ in $\mathbf{C}$, for $i, j=1, \ldots, N_{\alpha}$, and therefore, the net $\left(\pi_{\alpha}\left(\tau_{n}\right)\right)$ converges to $\pi_{\alpha}(\tau)$ in $M_{N_{\alpha}}(\mathbf{C})$. Hence, $\left(\pi\left(\tau_{n}\right)\right)$ converges to $\pi(\tau)$ in the product topology of $\prod_{\alpha} M_{N_{\alpha}}(\mathbf{C})$. The proof that the inverse of $\pi$ is continuous, and therefore that $\pi$ is a homeomorphism, is equally easy.

We use the representation $\pi$ to set up a functional calculus in $\mathcal{A}^{\prime}$.

5.2. Theorem. Let $\tau \in \mathcal{A}^{\prime}$. Let $\mathcal{E}$ denote the algebra of entire functions, where the operations are the pointwise-defined ones, and endow $\mathcal{E}$ with the topology of uniform convergence on compact subsets of $\mathbf{C}$. Then there is a unique, unital, continuous homomorphism $\theta$ from $\mathcal{E}$ to $\mathcal{A}^{\prime}$ that maps the identity function $z$ of $\mathbf{C}$ onto $\tau$. If $f \in \mathcal{E}$ has power series expansion given by $f(\lambda)=\sum_{n=0}^{\infty} c_{n} \lambda^{n}$, for all $\lambda \in \mathbf{C}$, then $\theta(f)=\sum_{n=0}^{\infty} c_{n} \tau^{n}$.

Proof. Let $f \in \mathcal{E}$. By the usual analytic functional calculus, the $C^{*}$-algebra $M_{N_{\alpha}}(\mathbf{C})$ contains the element $f\left(\pi_{\alpha}(\tau)\right)$. We denote by $\theta(f)$ the unique element of $\mathcal{A}^{\prime}$ for which $\pi(\theta(f))=\left(f\left(\pi_{\alpha}(\tau)\right)\right)_{\alpha}$. Since the map, $f \mapsto f\left(\pi_{\alpha}(\tau)\right)$, is a continuous, unital homomorphism from $\mathcal{E}$ to $M_{N_{\alpha}}(\mathbf{C})$ mapping $z$ onto $\pi_{\alpha}(\tau)$, the map, $f \mapsto \pi(\theta(f))$, is a continuous, unital homomorphism from $\mathcal{E}$ to $\prod_{\alpha} M_{N_{\alpha}}(\mathbf{C})$ that sends $z$ onto $\pi(\tau)$. Hence, the map, $f \mapsto \theta(f)$, is a continuous, unital homomorphism from $\mathcal{E}$ to $\mathcal{A}^{\prime}$, since $\pi$ is a homeomorphic isomorphism of $\mathcal{A}^{\prime}$ onto $\prod_{\alpha} M_{N_{\alpha}}(\mathbf{C})$. Moreover, $\theta(z)=\tau$.

To complete this proof, suppose now that $\theta$ is any continuous, unital homomorphism from $\mathcal{E}$ to $\mathcal{A}^{\prime}$ mapping $z$ to $\tau$. Let $f \in \mathcal{E}$ have power series representation $f(\lambda)=\sum_{n=0}^{\infty} c_{n} \lambda^{n}$, for all $\lambda \in \mathbf{C}$. Set $p_{n}=\sum_{k=0}^{n} c_{k} z^{k}$. Then $\theta\left(p_{n}\right)=\sum_{k=0}^{n} c_{k} \theta(z)^{k}=\sum_{k=0}^{n} c_{k} \tau^{k}$. Since the sequence $p_{n}$ converges uniformly to $f$ on compact subsets of $\mathbf{C}$, the sequence $\left(\theta\left(p_{n}\right)\right)$ converges to $\theta(f)$ in $\mathcal{A}^{\prime}$. Hence, $\theta(f)=\sum_{n=0}^{\infty} c_{n} \tau^{n}$. This concludes the proof, since we now see uniqueness of the homomorphism $\theta$ and we also see that $\theta$ has the remaining properties required of it in the statement of the theorem.

If $f \in \mathcal{E}$ and $\tau \in \mathcal{A}^{\prime}$, we write $f(\tau)$ for $\theta(\tau)$. Thus, if $f$ has power series expansion $f(\lambda)=\sum_{n=0}^{\infty} c_{n} \lambda^{n}$, for all $\lambda \in \mathbf{C}$, then $f(\tau)(a)=\sum_{n=0}^{\infty} c_{n} \tau^{n}(a)$, for all $a \in \mathcal{A}$.

It is not clear to the authors how one could define the functional calculus directly, without using the representation $\pi$, for it is not, a priori, obvious that the series $\sum_{n=0}^{\infty} c_{n} \tau^{n}(a)$ is convergent (remember that $\tau$ is not assumed to be bounded).

If $\tau \in \mathcal{A}^{\prime}$, then $\exp (\tau) \in \mathcal{A}^{\prime}$ also, where $\exp (\tau)(a)=\sum_{n=0}^{\infty} \tau^{n}(a) / n$ ! for all $a \in \mathcal{A}$. The existence of exponentials of elements of $\mathcal{A}^{\prime}$ may be significant for the quantum theory, since it plays an important role in the classical theory.

\section{ACKNOWLEDGEMENT}

It is with pleasure that the authors express their thanks to J. Kustermans and A. Van Daele for useful conversations concerning the material of this paper. 


\section{REFERENCES}

1. E. Abe, Hopf Algebras, Cambridge University Press, Cambridge, 1980. MR 83a:16010

2. K. Abodayeh and G. J. Murphy, Compact topological semigroups, Proc. Royal Irish Acad. 97A (1997), 131-137. MR 99g:22002

3. E. Bedos, G. J. Murphy and L. Tuset, Co-amenability of compact quantum groups, J. Geom. Phys. 40 (2001), 130-153. MR 2002m:46100

4. E. Bedos, G. J. Murphy and L. Tuset, Amenability and coamenability of algebraic quantum groups, International J. Math. and Math. Sci. 31 (2002), 577-601. MR 2003j:46107]

5. V. G. Drinfeld, Quantum groups, Proceedings of the International Congress of Mathematicians, Berkeley (1986), 793-820. MR 89f:17017

6. M. Enock and J.-M. Schwartz, Kac Algebras and Duality of Locally Compact Groups, Springer, Berlin-Heidelberg, 1992. MR 94e:46001

7. C. Kassel, Quantum Groups, Springer, Berlin-Heidelberg, 1995. MR 96e:17041

8. E. C. Lance, Hilbert $C^{*}$-Modules - A Toolkit for Operator Algebraists, Cambridge University Press, Cambridge, 1995. MR 96k:46100

9. A. Maes and A. Van Daele, Notes on compact quantum groups, Nieuw Arch. Voor Wisk. 16 (1998), 73-112. MR 99g:46105

10. G. J. Murphy, $C^{*}$-Algebras and Operator Theory, Academic Press, San Diego, 1990. MR 91m:46084

11. A. Van Daele, The Haar measure on a compact quantum group, Proc. Amer. Math. Soc. 123 (1995), 3125-3128. MR 95m:46097

12. S. Wassermann, Exact $C^{*}$-Algebras and Related Topics, University Press, National University Seoul, 1994. MR 95b:46081

13. S. L. Woronowicz, Compact matrix pseudogroups, Comm. Math. Phys. 111 (1987), 613-665. MR 88m:46079

14. S. L. Woronowicz, Twisted $S U(2)$ groups - an example of a noncommutative differential calculus, Publ. RIMS Kyoto Univ. 23 (1987), 117-181. MR 88h:46130

15. S. L. Woronowicz, Compact quantum groups, in Symétries Quantiques, North Holland, Amsterdam, 1998, pp. 845-884. MR 99m:46164

Department of Mathematics, National University of Ireland, Cork, Ireland

Faculty of Engineering, University College, Oslo, Norway 